\title{
Improving Secret Key Generation Performance for On-Body Devices
}

\author{
Linjia $\mathrm{Yao}^{\dagger}$, Syed Taha Ali ${ }^{\dagger}$, Vijay Sivaraman ${ }^{\dagger}$, Diethelm Ostry $\ddagger$ \\ †School of Electrical Engineering and Telecommunications \\ University of New South Wales, Sydney, Australia \\ ¥ICT Centre, CSIRO, Sydney, Australia \\ \{linjia,taha\}@student.unsw.edu.au; vijay@unsw.edu.au; diet.ostry@csiro.au
}

\begin{abstract}
In this paper, we undertake experiments to assess the feasibility of generating common secret keys between two bodyworn devices using the near-body channel. Deriving secret keys using the wireless channel is a practical and lightweight alternative to public-key key-agreement approaches. Our results indicate that key generation is good for dynamic scenarios where communicating devices are placed in non-lineof-sight positions on the body. Furthermore, we enhance existing key generation mechanisms by proposing a filtering method to reduce bit mismatch.
\end{abstract}

\section{Categories and Subject Descriptors}

C.2.0 [COMPUTER-COMMUNICATION NETWORKS]: General-Security and protection

\section{General Terms}

Security, Experimentation, Measurement, Performance

\section{Keywords}

Secret Key Agreement, Body Area Networks, RSSI

\section{INTRODUCTION}

In the recent years there has been increasing application of body area networks for healthcare. In such applications, bodyworn wireless medical sensor devices measure the patient's vital signs such as heart rate, temperature, blood glucose level, etc. Since these devices collect and communicate medical data, there are stringent ethical and legal implications, and security of this data is a critical concern. However, due to severely constrained computational and energy resources, these miniature sensor devices are not suited to run resource-intensive security solutions such as those based on public key cryptography. Instead, some researchers are working on new lightweight alternatives for small devices.

The problem we examine in this paper is that of key generation. For secure communication, two parties need to be

Permission to make digital or hard copies of all or part of this work for personal or classroom use is granted without fee provided that copies are not made or distributed for profit or commercial advantage and that copies bear this notice and the full citation on the first page. To copy otherwise, to republish, to post on servers or to redistribute to lists, requires prior specific permission and/or a fee. BODYNETS 2011, November 07-08, Beijing, People's Republic of China Copyright @ 2012 ICST 978-1-936968-29-9

DOI 10.4108/icst.bodynets.2011.247062 in possession of a shared secret. The Diffie-Hellman key exchange is typically used for this purpose, but recent results have shown that it is resource and time consuming to perform on small devices. A promising alternative approach is to use the unique properties of the wireless channel between two communicating devices to generate secret keys [1].

Wireless communication is fundamentally insecure but the channel between two communicating parties is unique to them [2]. If one party, Alice, transmits to Bob, the signal traverses multiple paths, experiencing different degrees of attenuation and phase shift, and Bob receives the summation of these multipath signals. If Bob responds in the exact same conditions, Alice's impulse response would be highly correlated to Bob's. This correlated information can be used to generate a secret key. A third party, Eve, located at least half a wavelength away from either Alice or Bob, and listening in on all transmissions, is limited to measuring a different channel, and for a dynamic multipath environment, the impulse response would be very different and near-impossible to guess for the Alice-and-Bob channel.

In this work, we explore this approach for on-body networks and introduce a modification to improve performance. This paper makes the following contributions: (1) We assess the suitability of using the near-body wireless channel for key generation for various configurations of device placement on the body and different modes of activity, and (2) we suggest a filtering modification to reduce bit mismatch between two devices and validate results with simulation and experiment.

\section{BACKGROUND AND PRIOR WORK}

Secret key generation schemes in the literature typically comprise four stages: in the sampling phase, two communicating parties continuously exchange probe packets to estimate reciprocal channel state. These channel estimations are then converted to key bits in the quantization step. However, mostly due to small-scale noise effects (that are random and uncorrelated for both parties) some generated bits are liable to disagree between the two parties, and information reconciliation is used to correct the mismatch by exchange of feedback. Additionally some of the bits may be correlated and a transform operation, i.e. privacy amplification is used to minimize this advantage for an eavesdropper who may acquire knowledge of some key bits.

In [3], the authors describe a method to extract key bits from a statistical Gaussian channel. They validate this key generation mechanism using the 802.11 platform, and gener- 

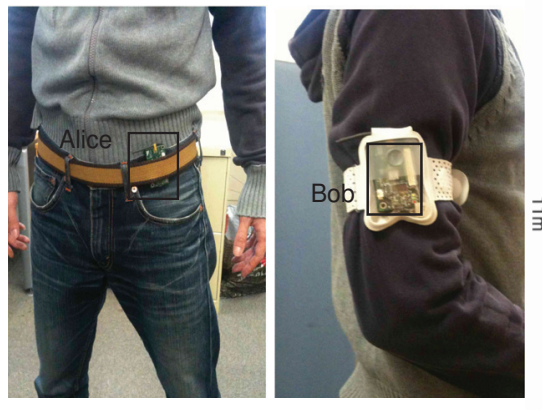

(a)

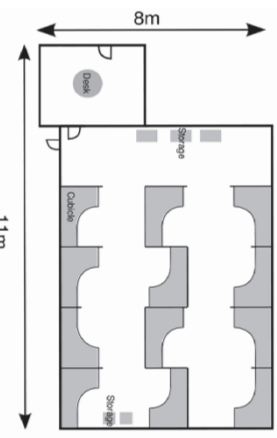

(b)
Figure 1: (a)Bodyworn sensors mounted on male (weight $58 \mathrm{~kg}$ and height $1.75 \mathrm{~m}$ ) : (Left) Alice on subject's left waist, (Right) Bob on subject's right arm. (b)Layout of office environment

ate bits at a rate of $1 \mathrm{bit} / \mathrm{sec}$ with nearly perfect secret key agreement in an indoor wireless environment. This approach is modified in [2], where the authors gather empirical measurements of received signal strength (RSS) for various environments with laptops using the wireless $802.11 \mathrm{~g}$ standard. Their scheme aims at generating high entropy secret bits at faster rates. In [4], the authors use a decorrelation technique and muti-bit quantization to achieve 40 secret bits/s with a $4 \%$ secret bit disagreement using TelosB motes.

In [5], we demonstrate an RSS-based secret key generation scheme for body area networks (sensor device on-body and basestation off-body) where the Savitzky Golay filter is used to isolate components, extracting secret keys at rate of about $1.25 \mathrm{bits} / \mathrm{s}$ with nearly perfect secret key agreement using slow component. In [6], the authors examine the near-body radio channel (with both devices on the body) for key generation. Their results, based on simulation modeling, indicate a potential key generation rate of about $2 \mathrm{bits} / \mathrm{s}$. However, they do not perform actual key generation and experimental observations do not involve mobility.

We believe ours is the first work to investigate key generation for a fully body-worn scenario and involves patient mobility, different placement of devices on the body, and different modes of motion such as resting and walking.

\section{FEASIBILITY OF KEY GENERATION IN ON-BODY NETWORK}

\subsection{Experimental Setup}

We performed experiments in an indoor office space consisting of multiple cubicles, as shown in Fig.1(b). We use MicaZ motes running TinyOs, operating in the $2.4 \mathrm{GHz}$ band. Two communicating devices, Alice and Bob, are mounted on the body $(1(\mathrm{a}))$, and transmit probe packets at $0 \mathrm{dBm}$ power at a rate of 50 packets/s, and sample the channel continuously. Two passive eavesdroppers are present: Eve1 is also mounted on the subject's body, and Eve2 is off-body

We collect RSS traces for four scenarios listed in Table 1, categorized by subject's activity (Walking or Resting) and placement of devices on body. For walking scenarios the subject walks randomly around the office at about $1 \mathrm{~m} / \mathrm{s}$. Readings are collected over a span of $3 \sim 5$ minutes.
Table 1: Experimental Setup

\begin{tabular}{|c|c|c|c|c|c|}
\hline Scenario & $\begin{array}{c}\text { Alice's } \\
\text { Location }\end{array}$ & $\begin{array}{c}\text { Bob's } \\
\text { Location }\end{array}$ & $\begin{array}{c}\text { Eve1's } \\
\text { Location }\end{array}$ & $\begin{array}{c}\text { Eve2's } \\
\text { Location }\end{array}$ & $\begin{array}{c}\text { User's } \\
\text { Motion }\end{array}$ \\
\hline a & L Waist & R Arm & L Arm & Desk & Resting \\
\hline b & L Waist & R Arm & L Arm & Desk & Walking \\
\hline c & L Waist & R Leg & L Arm & Desk & Walking \\
\hline d & L Waist & L Chest & L Arm & Desk & Walking \\
\hline
\end{tabular}

\subsection{Experimental results}

Fig. 2 shows RSS traces for the four scenarios. For clarity, we only show readings over a period of 10 seconds and eavesdroppers' measurements are only plotted for Scenario (b) in Table 1. As can be seen, the channel state variation is very dependent on mode of activity and device placement. We make the following observations:

- During resting (Scenario (a)), the channel state has minimal variation, and the correlated information that can be extracted for bit generation will be very low.

- For Scenarios (b) and (c), the transmission between Alice and Bob is non-light-of-sight (NLOS) due to antenna placement and because the subject is moving, there is significant channel variation which is excellent for key generation.

- For Scenario (d), the antennas for Alice and Bob have line of sight(LOS) due to placement on the chest and waist, and their distance is roughly constant. The channel variation is much smaller compared to Scenario (b) and (c). We suggest that this is because the line of sight ensures that the RSS is dominated by the path loss component which is relatively steady in this case, whereas for NLOS scenarios (b) and (c), multipath propagation dominates the channel variation.

- Eavesdroppers, due to their placement, measure a different channel and their results are uncorrelated to those of Alice and Bob (Fig. 2(e)).

We employ the Pearson correlation coefficient to estimate the correlation for different parties. A value of 1 indicates perfect correlation, 0 denotes no correlation and -1 implies anticorrelation. For dynamic scenarios, the RSS correlation between Alice and Bob for LOS (Scenario (d)) is 0.8513 and for NLOS (Scenarios (b) and (c)), it is higher at 0.9825 and 0.9831 . The higher the correlation, the more likely the resulting keys are to agree between Alice and Bob.

Existing research [3] indicates that small-scale effects negatively affect the correlation. These are due to uncorrelated white noise at both endpoints, and asynchronous sampling. Most radios have half-duplex modes of communication which do not permit simultaneous sampling of the channel by both endpoints. Instead both devices sample the channel in quick succession and in this time interval, the channel is likely to vary by a small amount. To minimize these small-scale effects for better bit agreement, we propose filtering using an averaging technique.

\section{FILTERING TO IMPROVE KEY MATCH}

\subsection{Filtering: idea and analysis}

To convert RSS measurements to secret key bits, we adapt a quantizer originally proposed by Mathur et al.[3] and modified by Jana et al.[2]. In Mathur's quantization scheme, two 
(a) Resting LWaist-RArm

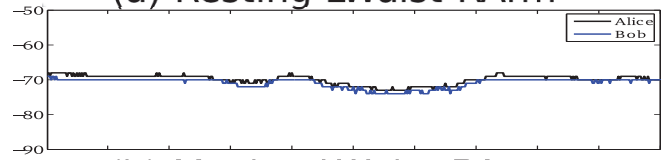

(b) Moving LWaist-RArm

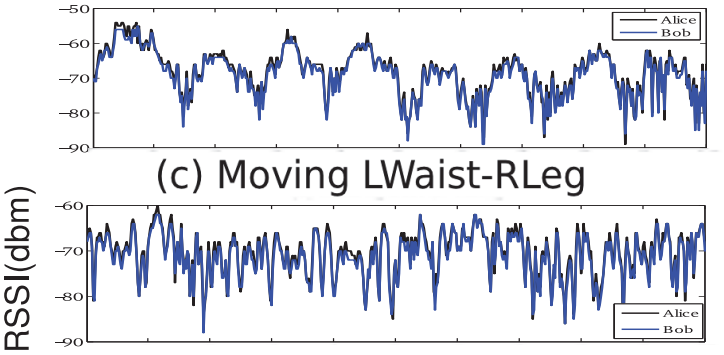

(d) Moving LWaist-LChest

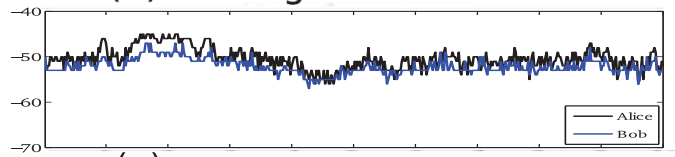

(e) Moving LWaist-RArm

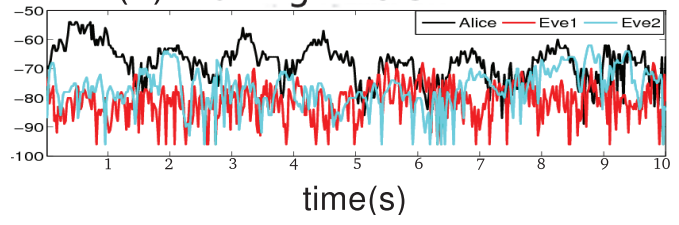

Figure 2: 10 seconds RSS measurements

thresholds $q+$ and $q-$ are determined over a block of readings of size $f$ such that:

$$
q+=\mu+\alpha \cdot \sigma \quad ; \quad q-=\mu-\alpha \cdot \sigma
$$

where $\mu$ and $\sigma$ denote mean and standard deviation of the sampling values and $\alpha$ is a configurable parameter such that $\alpha \geq 0$. If $m$ successive values lying above $q+$ or below $q-$ are encountered, a single bit 1 or 0 are encoded, respectively. Samples lying within the thresholds are dropped.

In our modified quantizer (depicted in Fig. 3), we still calculate the $q$ thresholds using Eq.(1), but we perform averaging of the sample values over a window size $w$ to minimize the small-scale uncorrelated effects for both parties. Instead of waiting for $m$ successive values, if any resulting value is greater than or less than the $q+$ or $q$ - respectively, we encode to a 1 or a 0 . In this case, block size $f=n \cdot w$.

We provide a theoretical justification of our approach: assuming Gaussian estimation of channel state and independent successive sampling values, the secret bit mismatch $\left(p_{b m}\right)$ and secret bit rate $\left(R_{s b}\right)$ of the quantization result are expressed in Eqs.(2) and (3).

$$
\begin{aligned}
p_{b m} & =\frac{P(A=1, B=0)}{P(A=1, B=0)+P(A=1, B=1)} \\
& =\frac{\int_{-\infty}^{-q} \int_{+q}^{\infty} f\left(x_{i}, y_{i}\right) d x_{i} d y_{i}}{\int_{-\infty}^{-q} \int_{+q}^{\infty} f\left(x_{i}, y_{i}\right) d x_{i} d y_{i}+\int_{+q}^{\infty} \int_{+q}^{\infty} f\left(x_{i}, y_{i}\right) d x_{i} d y_{i}}(2) \\
R_{s b} & =\frac{f_{s}}{w} \cdot[P(A=0, B=0)+P(A=1, B=1)] \\
& =\frac{f_{s}}{w} \cdot 2 \int_{+q}^{\infty} \int_{+q}^{\infty} f\left(x_{i}, y_{i}\right) d x_{i} d y_{i}
\end{aligned}
$$

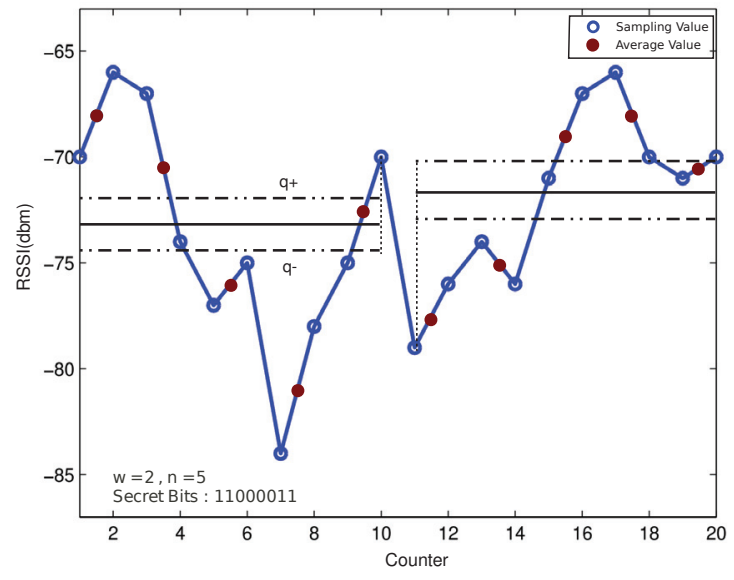

Figure 3: A sample of our quantization with $w=2$ and $n=5$.

where

$$
\begin{gathered}
f\left(x_{i}, y_{i}\right)=\frac{1}{2 \pi \sigma_{x_{i}} \sigma_{y_{i}} \sqrt{1-\rho^{2}}} e^{-\frac{1}{2\left(1-\rho^{2}\right)} z}, \\
z \equiv \frac{\left(x_{i}-\mu_{x_{i}}\right)^{2}}{\sigma_{x_{i}}^{2}}+\frac{\left(y_{i}-\mu_{y_{i}}\right)^{2}}{\sigma_{y_{i}}^{2}}-\frac{2 \rho\left(x_{i}-\mu_{x_{i}}\right)\left(y_{i}-\mu_{y_{i}}\right)}{\sigma_{x_{i}} \sigma_{y_{i}}} .
\end{gathered}
$$

Here $f_{s}$ is the maximum sampling rate needed to derive independent sampling values, and $x_{i}$ and $y_{i}$ are the average of sampling values within a window for Alice and Bob respectively, with correlation $\rho$.

We present analytical and simulation results in Fig. 4(a) and $4(\mathrm{~b})$ and observe that when window size increases, bit match improves as was our motivation. However, a tradeoff is seen in that the averaging results in a reduction in secret bit rate. This is because the number of samples effectively fed into the quantizer are now reduced due to the averaging and therefore less bits will be produced.

\subsection{Filtering in practice}

We validate our approach using RSS traces from dynamic Scenario (b), (c) and (d) in our experiments. To compare performance of our modified quantization with that of Mathur and Jana, we set identical quantization parameters for all scenarios. According to [7], the state of the dynamic body area channel is steady for 5-25 ms. Since our devices sample the channel every $20 \mathrm{~ms}$, we therefore set $w$ to the minimum $w=2$ to ensure that successive secret bits are uncorrelated. We set block size $f=20$ samples, i.e. quantization is done approximately every $200 \mathrm{~ms}$. Parameter $\alpha$ allows us to tweak the tradeoff between secret bit rate and bit mismatch and for our purposes $\alpha=0.5$. The results are shown in Table 2. We make the following observations:

- Even though eavesdroppers receive all packets exchanged by Alice and Bob and use the same quantization parameters, because they are fundamentally sampling a different channel, they encounter approximately $50 \%$ bit mismatch which is equivalent to randomly guessing the secret key agreed upon by Alice and Bob. 


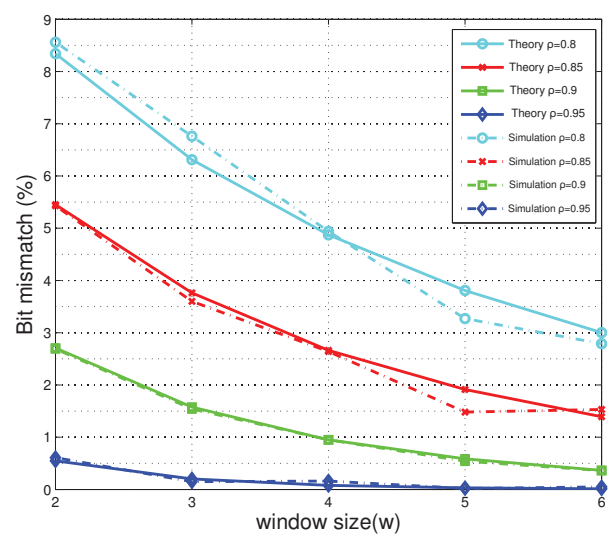

(a) Bit mismatch against window size for different correlation values

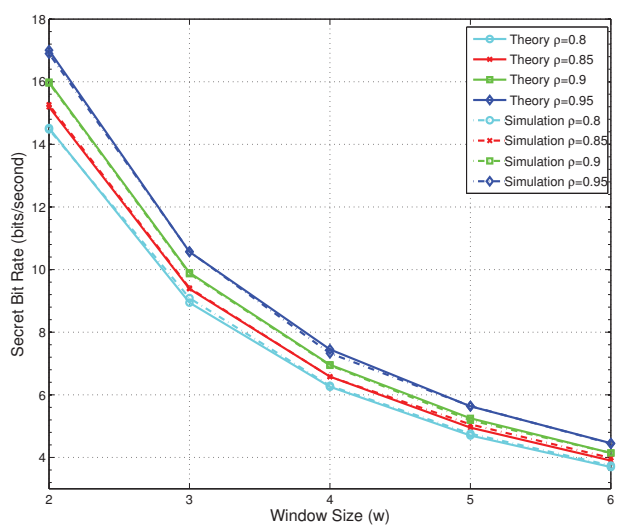

(b) Secret bit rate against window size for different correlation values

Figure 4: Analytical and simulation results, $\alpha=0.2$ and $f_{s}=50 \mathrm{~Hz}$

- Secret key bits are generated at a high rate with a low secret bit mismatch for NLOS links. We noted earlier that there was high correlation between Alice and Bob for NLOS links.

- Key bit mismatch is higher for the LOS link. We predicted this when we computed correlation for the different links in Section 3.2. In this case, increasing window size incrementally will reduce bit mismatch.

We use the last instance, the LOS link to compare the performance of our modified quantizer with that of Mathur and Jana, since that shows the highest bit mismatch. Results are depicted in Table 3. The individual parameters for the different quantizers are set as shown such that they yield equivalent secret bit rate $(3.75 \sim 3.85 \mathrm{bits} / \mathrm{s})$. We observe that our quantizer performs best by giving the lowest bit mismatch of all three, thereby validating our approach.
Table 2: Experimental Results for Moving Scenarios: $w=$ $2, n=5, \alpha=0.5$

\begin{tabular}{|c|c|c|c|c|}
\hline Valid Mote & Secret Bit & \multicolumn{3}{|c|}{ Secret Bit Mismatch } \\
\cline { 3 - 5 } Location & Rate(bits/s) & Valid Mote & Eve1 & Eve2 \\
\hline $\begin{array}{c}\text { L Waist } \\
\text { R Arm(NLOS) }\end{array}$ & 13.10 & $0.13 \%$ & $52.69 \%$ & $47.85 \%$ \\
\hline $\begin{array}{c}\text { L Waist } \\
\text { R Leg(NLOS) }\end{array}$ & 13.90 & 0 & $49.10 \%$ & $48.84 \%$ \\
\hline $\begin{array}{c}\text { L Waist } \\
\text { L Chest(LOS) }\end{array}$ & 8.42 & $7.37 \%$ & $50.32 \%$ & $51.62 \%$ \\
\hline
\end{tabular}

Table 3: Experimental Result: LWaist-LChest

\begin{tabular}{|c|c|c|}
\hline Quantization & $\begin{array}{c}\text { Secret Bit } \\
\text { Rate(bits/s })\end{array}$ & $\begin{array}{c}\text { Valid Mote } \\
\text { Mismatch }\end{array}$ \\
\hline $\begin{array}{c}\text { Mathur } \\
(\mathrm{m}=2, \alpha=0.54)\end{array}$ & 3.81 & $2.73 \%$ \\
\hline $\begin{array}{c}\text { Jana } \\
(\alpha=1.18, \text { blocksize }=12)\end{array}$ & 3.76 & $5.13 \%$ \\
\hline $\begin{array}{c}\text { Ours } \\
(\mathrm{w}=4, \mathrm{n}=3, \alpha=0.5)\end{array}$ & 3.84 & $1.46 \%$ \\
\hline
\end{tabular}

\section{CONCLUSION}

In this paper we have demonstrated the feasibility of extracting secret keys from the wireless channel in on-body networks. Our experimental results confirm that human motion causes high channel variation to generate secret keys at a fast rate with high agreement, especially for NLOS links. Eavesdroppers are unable to replicate the secret key even if they are placed within a moderate distance of the communicating parties. Furthermore, we enhance existing quantization techniques with a filtering function to minimize random noise effects and improve secret key agreement. For future work, we intend to optimize quantizer design and address the issue of authentication of bodyworn devices.

\section{REFERENCES}

[1] M. Wilhelm, I. Martinovic, and J. B. Schmitt. Secret keys from entangled sensor motes: implementation and analysis. In ACM WiSec, 2010.

[2] S. Jana, S. N. Premnath, M. Clark, S. Kasera, N. Patwari, and S. Krishnamurthy. On the effectiveness of secret key extraction from wireless signal strength in real environments. In ACM MobiCom, 2009.

[3] S. Mathur, W. Trappe, N. Mandayam, C. Ye, and A. Reznik. Radio-telepathy: extracting a secret key from an unauthenticated wireless channel. In $A C M$ MobiCom, 2008.

[4] J. Croft, N. Patwari, and S. Kasera. Robust uncorrelated bit extraction methodologies for wireless sensors. In $A C M / I E E E$, IPSN, 2010.

[5] S. T. Ali, V. Sivaraman, and D. Ostry. Secret key generation rate vs. reconciliation cost using wireless channel characteristics in body area networks. In IEEE Trustcom, 2010.

[6] L. W. Hanlen, D. Smith, J. Zhang, and D. Lewis. Key-sharing via channel randomness in narrowband body area networks: is everyday movement sufficient? In Bodynets, 2009.

[7] J. Zhang, D. B. Smith, L. W. Hanlen, D. Miniutti, D. Rodda, and B. Gilbert. Stability of narrowband dynamic body area channel. Antennas and Wireless Propagation Letters, IEEE, 2009. 